\title{
EL (INCOMPLETO Y BREVE) REGRESO DE LA POLÍTICA INDUSTRIAL: EL CASO DE ARGENTINA 2003-2015
}

\author{
Pablo Lavarello
}

Fecha de recepción: 14 de noviembre de 2016. Fecha de aceptación: 08 de marzo de 2017.

\begin{abstract}
RESUMEN
En un contexto de expansión de la economía mundial, ampliación de brechas tecnológicas y una evolución de precios relativos adversos a la industrialización, Argentina se encuentra entre los países de América Latina en los que la política industrial regresa (en forma parcial y transitoria) a la agenda de política económica. En este trabajo se plantea como hipótesis que pese al regreso de acciones e instrumentos de política industrial que promueven las capacidades tecnológicas y generan un marco de incentivos favorables a la industrialización, las mismas no llegaron a configurar una nueva política industrial dada la baja coordinación y la ausencia de una instancia jerárquica superior de implementación.
\end{abstract}

Palabras clave: política industrial, industrialización, capacidades tecnológicas, planificación, incentivos fiscales y financieros.

Clasificación JEL: L16, L52, O14, O25.

\section{The (InCOMPlete And Brief) Return of Industrial Policy: The Case of Argentina 2003-2015}

\begin{abstract}
Against the backdrop of global economic growth, the widening of technology gaps, and the adverse evolution of prices for industrialization, Argentina is one of the countries in Latin America that has seen the (partial and temporary) return of public policy to the economic policy agenda. This paper poses the hypothesis that in spite of the revival of industrial policy actions and instruments to promote technological capabilities and foster a framework of incentives favorable to industrialization, a new industrial policy has failed to emerge in light of weak coordination and the absence of a senior body in the hierarchy to implement it.
\end{abstract}

Key Words: Industrial policy, industrialization, technological capabilities, planning, fiscal and financial incentives.

1 Centro de Estudios Urbanos Regionales del conicet y Universidad Nacional de San Martín, Argentina. Correo electrónico: plavarello@conicet.gov.ar 
LE (INCOMPLET ET BREF) RETOUR DE LA POLITIQUE INDUSTRIELLE: LE CAS DE L'ARGENTINE 2003-2015

\section{Résumé}

Dans un contexte d'expansion de l'économie mondiale, extension des écarts technologiques et d'une évolution des prix relatifs défavorables à l'industrialisation, l'Argentine se classe parmi les pays d'Amérique Latine où la politique industrielle revient (sous une forme partielle et transitoire) à l'agenda du politique économique. Dans cet article, nous avons émis l'hypothèse que, malgré le retour des actions et des instruments de politique industrielle qui favorisent les capacités technologiques et qui créent un cadre favorable aux incitations à l'industrialisation, elles ont échoué à définir une nouvelle politique industrielle compte tenu de la faible coordination et absence d'une instance de déploiement supérieur hiérarchique.

Mots clés: politique industrielle, industrialisation, capacités technologiques, planification, incitations fiscales et financières.

\section{O RETORNO (INCOMPLETO E BREVE) DA POLÍTICA INDUSTRIAL: O CASO DA ARGENTINA 2003-2015 \\ Resumo}

Num contexto de expansão da economia mundial, de expansão das brechas tecnológicas e de uma evolução de preços relativos adversos à industrialização, Argentina está entre os países latino-americanos nos quais a política industrial tinha regressado (de forma parcial e transitória) para agenda de política económica. Neste trabalho, a hipótese que se formula infere que apesar do retorno das açóes e instrumentos de política industrial que promovem capacidades tecnológicas e criam um quadro favorável de incentivos á industrialização, eles não conseguiram definir uma nova política industrial, dada a fraca coordenaçáo e a ausência de uma instancia hierárquica superior para sua implementação.

Palavras-chave: política industrial, industrialização, capacidades tecnológicas, planejamento, incentivos fiscais e financeiros.

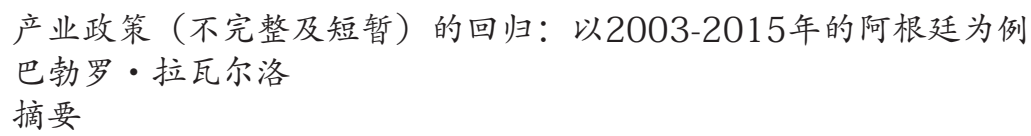
并促进了产业化刺激机制的形成, 由于低效的配置及高效层级式实施机制 的缺乏, 这些产业政策并没能最终促成新产业政策的出现。 关键词：产业政策、产业化、技术能力、规划、财政及金融刺激 


\section{INTRODUCCIÓN ${ }^{2}$}

El objetivo de este artículo es discutir el regreso de la política industrial a la agenda de política económica en América Latina durante la primera década de los años 2000. El caso de Argentina es de amplia relevancia dado que es uno de los países de la región en los cuales este regreso se manifiesta después de décadas de ausencia.

Hacia mediados de los años setenta del siglo xx, la economía argentina había llegado a ser la economía de América Latina con la estructura industrial relativamente más diversificada y menos heterogénea como resultado de un largo proceso de industrialización. Luego, dicho país experimentó entre el fin de los años setenta y noventa uno de los procesos de cambio estructural regresivo más fuertes que se produjeron a nivel internacional, al adoptar un conjunto de políticas de liberalización y apertura que llevaron a la destrucción de las capacidades tecnológicas (e institucionales) acumuladas durante la posguerra.

La política industrial reaparece en Argentina durante los años 2000, no como resultado de una asimilación de experiencias pasadas, sino como necesidad de dar respuesta a los problemas de escasez de divisas propios de un proceso de crecimiento impulsado por la demanda (Abeles et al., 2013). Es así que luego de cinco años de crecimiento hacia el año 2008, esta necesidad se vio reforzada por un lado, frente el estrangulamiento en la disponibilidad de divisas (los mencionados ciclos de "parada y arranque"), hecho que se profundizaría en el año 2013 con la caída en los términos de intercambio de las materias primas y una nueva fase de contracción de la economía mundial que se tradujo en la sobreoferta de productos importados.

Cabe interrogarse, por un lado, si estas respuestas frente a los cambios en las condiciones internas y externas se tradujeron en el pasaje de una modalidad de intervención inspirada en la concepción de Estado facilitador que limita su injerencia a la resolución de "fallas de mercado" o asumió el carácter de una política industrial en el sentido estricto, como un conjunto coordinado de acciones sobre las capacidades tecnológicas y los incentivos orientados a generar

\footnotetext{
Este artículo discute desde una perspectiva crítica un conjunto de resultados empíricos obtenidos en trabajos previos del autor, en particular en Lavarello, P. J. y Sarabia, M. (2015), "La política industrial en la Argentina durante la década de 2000”, Serie Estudios y Perspectivas, núm. 45, Cepal. Se agradecen los comentarios y sugerencias a dicho documento en numerosos seminarios a una versión previa en co-autoría con Matias Mancini (2017). Igualmente la sistematización de los resultados y las conclusiones desarrollados en esta versión son de responsabilidad exclusiva del autor.
} 
un proceso de cambio estructural desafiando (en mayor o menor grado) las ventajas comparativas.

Buscando responder a este interrogante, el presente artículo se organiza de la siguiente manera: en la sección 1 se discute brevemente el tratamiento que se ha dado desde la teoría económica a la política industrial; en la sección 2 se plantea un marco conceptual para el análisis de las distintas acciones e instrumentos de política industrial. Se presentan en forma abreviada los antecedentes de política industrial en Argentina a lo largo de la sección 3; se muestra en la sección 4 un análisis a partir de esta grilla conceptual de las principales acciones e instrumentos vigentes buscando identificar los cambios en los patrones de intervención sobre la industria en el periodo 2003-2015. Finalmente, se presentan las experiencias de planificación de la política industrial en el periodo en la sección 5 , y se concluye con la discusión de los principales aspectos analizados y con algunas enseñanzas generales de la experiencia de política industrial en dicho periodo.

\section{LA POLÍTICA INDUSTRIAL: DE LAS FALLAS DE MERCADO A LA GENERACIÓN DE CAPACIDADES}

Si existe un terreno en el que, salvo excepciones, se revela la ausencia de reconocimiento de la política industrial es en la teoría económica. El legado de Alexander Hamilton (1791) y Friedrich List (1841), que plantearon sus fundamentos, quedó limitado a un conjunto acotado de economistas "herejes" que circularon en los márgenes (o por fuera) de la corriente principal de la teoría económica. Los importantes aportes realizados por estos economistas tuvieron como impulso los desafíos que planteaba la industrialización tardía más que la curiosidad del investigador.

Durante los últimos años, se ha adoptado el enfoque de "fallas de mercado" para justificar las denominadas políticas de "desarrollo productivo" (Hausmann \& Rodrik, 2002; Lin \& Monga, 2011; Lin, 2012; Crespi, Fernández-Arias \& Stein, 2014). Desde esta perspectiva, además de proveer bienes públicos (tales como reglas, estándares o I\&D), las externalidades de información son vistas como la principal falla de mercado para la diversificación industrial para los países en desarrollo. Hausmann y Rodrik sostienen que una firma que entra en una nueva industria genera una externalidad al resto de los potenciales entrantes, en tanto, genera información nueva sobre la viabilidad de la nueva actividad y no es compensada por el riesgo que asume. 
En ese marco se justifican subsidios selectivos a estas firmas bajo la forma de créditos o garantías. En línea con este argumento, Justin Lin reconoce que es necesario que las economías en desarrollo se diversifiquen hacia aquellos productos y servicios transables que produzcan países desarrollados "con estructuras de dotaciones factoriales similares y con ingreso per cápita alrededor de $100 \%$ superior a las propias" (Lin, 2012, p. 161).

Más allá de la arbitrariedad del porcentaje de $100 \%$ de diferencia en el ingreso per cápita, los cambios en la estructura productiva deben ser graduales, ya que se limitan a externalidades de información dentro de la misma industria o a actividades con similares dotaciones factoriales. En ese marco, el Estado debe minimizar los costos de definir una mala especialización asumiendo un rol de "facilitador" del desarrollo sin desafiar el principio de las ventajas comparativas.

En un trabajo colectivo, junto a autores evolucionistas, Joseph Stiglitz -uno de los principales impulsores del abordaje de fallas de mercado-sostiene que no es que no existan fallas de mercado, sino que el mundo está plagado de las mismas (Cimoli, Dosi, Nelson \& Stiglitz, 2006). Se podría reforzar el argumento,si se tiene en cuenta que la dinámica de una economía capitalista se basa justamente en la generación endógena de fallas de mercado: la competencia a través de la introducción de nuevos productos y procesos.

Frente a esta situación de ausencia de una teoría operativa de política industrial, un conjunto de trabajos recientes de inspiración evolucionista y neoschumpeteriana (Lall, 1992; Cimoli et al., 2006; Cimoli, Ferraz, Primi, 2009) renuevan las hipótesis originales de la industria naciente de List y Hamilton haciendo hincapié en las diferentes habilidades entre los países en desarrollo para usar y desarrollar tecnología -o lo que estos trabajos denominan las "capacidades tecnológicas”- y, particularmente, los altos costos de adopción y absorción de la tecnología importada. Desde esta perspectiva, las capacidades tecnológicas e institucionales son el resultado de procesos de aprendizaje y, por lo tanto, son endógenas a los cambios en la estructura productiva.

El hecho de que la tecnología cuente con un gran componente tácito resultante de la experiencia (learning by doing) hace que sea muy difícil subir la "escalera de la industrialización" gradualmente a partir de las ventajas comparativas iniciales. Dichos aprendizajes no se limitan a la firma, sino que están enraizados en la estructura productiva. Existen ciertas industrias "ingeniería intensivas" como los proveedores especializados que cuentan con un mayor potencial de aprendizaje y, por lo tanto, de rendimientos crecientes externos (Pavitt, 1984; Bell \& Pavitt, 1995). En este sentido, estas actividades asumen un papel nodal en la difusión intersectorial de tecnología, entre ellas, destaca 
la industria de bienes de capital, que junto a las industrias de software sigue constituyendo la principal actividad difusora de tecnología (Bell \& Pavitt, 1995).

El desarrollo de estos sectores no es inducido endógenamente, en la medida que los precios relativos no revelan las oportunidades tecnológicas futuras. En periodos de cambio de paradigma tecnológico, en el que los costos comparativos se modifican drásticamente, el problema es aún mayor dada la incertidumbre radical, y la política industrial debe recurrir a acciones deliberadas de generación de nuevos sectores.

Esta visión tiene importantes implicaciones a la hora de abordar el diseño y la implementación de la política industrial, en la medida en que exige coordinar un conjunto de acciones en forma selectiva y deliberada a nivel tanto de la generación de capacidades tecnológicas como de los incentivos con el fin de lograr el cambio estructural.

\section{MARCO CONCEPTUAL PARA EL ANÁLISIS DE LA POLÍTICA INDUSTRIAL}

A partir de estas consideraciones generales es posible construir a partir de Lavarello y Mancini (2017) una matriz exploratoria de áreas y de niveles de intervención consistentes con distintas configuraciones institucionales de la política industrial (véase figura 1). En las columnas es posible identificar las principales áreas de intervención que surgen de un abordaje de las capacidades infantiles basadas en la experiencia histórica de distintas experiencias de catching up (Cimoli et al., 2006; Peres \& Primi, 2009; Evans, 1995).

Las áreas de intervención incluyen por un lado, todas aquellas acciones que usualmente se asocian a la política tecnológica y que actúan sobre las oportunidades de la Ciencia y la Tecnología a partir del financiamiento de la Investigación y Desarrollo ( $\mathrm{I}+\mathrm{D})$, aquellas que buscan reforzar la educación técnica y un conjunto de incentivos a la generación de capacidades tecnológicas en las firmas que permiten acelerar aprendizaje tecnológico; por el otro, todas aquellas acciones de política industrial que a través de distintos tipos de instrumentos -arancelarios, fiscales, de compra gubernamental, financieros, propiedad intelectual, regulatorios- afectan selectivamente las señales económicas y/o los contextos de selección de las firmas.

Estas áreas de intervención de la política industrial pueden presentar distinto alcance en términos de selectividad de las políticas, actuando a distintos niveles: a) aquellas que operan a nivel macroeconómico y del marco regula- 
torio general teniendo como objetivo generar condiciones generales sobre la economía (por ejemplo, reglas de financiamiento de la inversión, defensa de la competencia); b) instrumentos microeconómicos horizontales que buscan afectar directamente el comportamiento de las firmas a través de distintos incentivos (fiscales o financieros a la innovación o inversión sin discriminar sector o firma); c) instrumentos regionales, los que a través de políticas muy diferentes que van desde los tradicionales regímenes de promoción regionales o las políticas de clúster buscan favorecer ex ante el desarrollo de ciertas regiones; $d$ ) instrumentos selectivos que plantean desde su diseño una clara focalización y priorización a nivel de tecnología, sector o incluso selección de firma en el marco de la promoción de campeones nacionales; $e$ ) instrumentos selectivos y estructurantes, que se orientan un conjunto de acciones en grandes proyectos orientados por misiones con potencial de traccionar al resto de la economía.

Las distintas combinaciones de áreas de intervención y alcance de los instrumentos pueden articularse en configuraciones diferentes de política analizadas por la literatura (Johnson, 1982; Evans, 1995). En primer lugar, la configuración de intervención del "Estado regulador" señalada por Johnson y que corresponde con la visión que prevalece en el Congreso de los Estados Unidos, ${ }^{3}$ en la que el Estado se limita a establecer condiciones regulatorias generales a través de instrumentos de alcance macroeconómico u horizontal, sin ningún tipo de acción sobre las capacidades tecnológicas más allá de algún instrumento de incentivo horizontal a la $\mathrm{I}+\mathrm{D}$.

En contraste, es posible identificar configuraciones de intervención en línea con la idea de la industria naciente inspirada en el legado de F. List que combina instrumentos orientados a la generación de capacidades tecnológicas en las firmas con un marco regulatorio y de incentivos altamente selectivo. Esta configuración es consistente con las experiencias francesas de política industrial hasta los años ochenta (Cohen, 2007), y con la visión de "Estado desarrollista" de Johnson (1982) a partir de su estudio la experiencia de Japón luego ampliada a los estudios de las experiencias de Corea y Taiwán (Amsden, 1992; Evans, 1995).

Por último, en la medida que muchos de estos países cierran sus brechas tecnológicas surge una tercer modalidad de intervención que Evans (1995)

Según varios autores esta visión es parcial y no tiene en cuenta el rol intervencionista altamente selectivo que prevalece en la política industrial de Estados Unidos a través de la compra gubernamental y de grandes programas implementados desde el Departamento de Defensa (Wade, 2014; Mazzucato, 2013). 
denominó como aquella del "jardinero", en la que el Estado sin cuestionar los "precios de mercado" asume un rol facilitador intentando favorecer las iniciativas de las empresas -las "flores" del jardín- sin desafiar las ventajas comparativas.

Cada una de estas modalidades de intervención prevé distintos tipos de arreglos institucionales que a través de mecanismos de mercado, en red o jerárquicos buscan resolver problemas de coordinación frente a las complementariedades de inversión intersectoriales, el tipo de relación entre finanzas e industria, las interacciones de proveedores y usuarios.

La existencia de un conjunto de acciones orientado a promover la industria no significa, por supuesto, que las políticas industriales sean necesariamente efectivas. Esta discusión se ha reavivado, desde los años ochenta y surgen nuevos argumentos que hacen hincapié, en los problemas de implementación. Es bien conocido el argumento según el cual, una elevada concentración de la propiedad de los recursos naturales puede bloquear los procesos de cambio estructural cuando éstos afectan negativamente los intereses de los propietarios de los mismos (Cimoli \& Rovira, 2008).

En ese sentido cobra relevancia el concepto de "autonomía enraizada" de Peter Evans (1995) que sugiere que una política industrial efectiva requiere, por un lado, un relativo grado de autonomía del Estado - entendido como el conjunto de agencias que intervienen en este caso en la implementación de la misma- y, por el otro, cierto grado de enraizamiento en relaciones institucionalizadas con los capitalistas. Mientras que el enraizamiento sin autonomía se traduce en conductas de búsquedas de renta y en la posibilidad de captura de las agencias por el sector privado, la autonomía sin imbricación da lugar al aislamiento dificultando la identificación de oportunidades y el seguimiento de la política.

Bajo estas condiciones de autonomía enraizada es posible avanzar en procesos de aprendizaje institucional en la implementación de política. Como sostienen Peres y Primi (2009) la mayor selectividad de los instrumentos de política dependerá, por un lado, de los recursos asignados y la sofisticación de los instrumentos que posee la agencia u organismo involucrado, y por el otro, de las capacidades institucionales para llevar adelante las políticas.

Es así que los países en los que la intervención se limita a la regulación, las capacidades institucionales y los recursos de las agencias de política industrial son menores, y son más proclives a llevar adelante políticas horizontales basadas en la provisión de bienes públicos a partir de un conjunto limitado de instrumentos (asistencia técnica, cumplimiento de normas sanitarias y de salud, infraestructura de Ciencia y Tecnología (сут), formación de recursos 
Figura 1. Matriz de política industrial y tecnológica

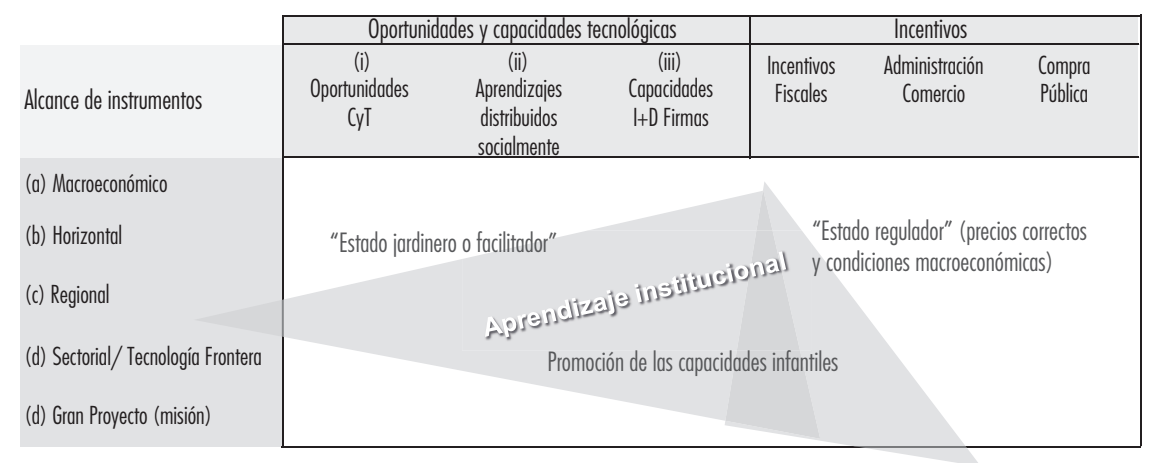

Fuente: elaboración propia con base en Lavarello y Mancini (2017).

humanos). A medida que se desarrollan capacidades institucionales de las agencias involucradas es posible avanzar hacia políticas propias de un "Estado facilitador o jardinero", y sólo cuando las capacidades de monitoreo, los recursos involucrados y la jerarquía de las agencias involucradas en el Estado son lo suficientemente elevados es posible implementar una intervención desarrollista de promoción de las capacidades infantiles con apoyo a sectores (y firmas) específicos a partir de grandes programas estructurantes (véase figura 1).

Aspectos que plantean un dilema para la política industrial: por un lado, las políticas industriales sectoriales requeridas para salir del subdesarrollo exigen contar con capacidades institucionales que, en caso que se las tuviera, ya no se trataría de un país subdesarrollado. En este sentido, ascender por la curva de los aprendizajes institucionales no es un proceso lineal y armónico y requiere, tal como seńalaba Albert Hirschman (1958) generar, en ciertos momentos, efectos desequilibrantes de política que despierten una secuencia de eslabonamientos y aprendizajes institucionales.

En las secciones siguientes intentaremos ilustrar cuál es la trayectoria de aprendizaje institucional de dichas políticas interrogándonos si se avanzó tal como indica el área gris de la figura 1 desde una concepción de Estado regulador a una configuración en el que los instrumentos de apoyo a las capacidades tecnológicas, los incentivos fiscales y los marcos regulatorios asumieron una mayor variedad de instrumentos al mismo tiempo que ganaban selectividad y estructuración en grandes proyectos. 


\section{ANTECEDENTES DE POLÍTICA INDUSTRIAL EN ARGENTINA}

Desde su conformación como Estado-nación, la Argentina no logró implementar un proceso deliberado de cambio estructural que desafíe las ventajas comparativas (Azpiazu \& Notcheff, 1995). La industrialización se fue generando en respuesta a "cuellos de botella" propios de la restricción de divisas y objetivos estratégicos ligados a la autonomía nacional (Katz \& Kosacoff, 1998). Las acciones de apoyo a la industrialización asumieron una mayor relevancia entre fines de los años sesenta y setenta con la promoción deliberada a sectores estratégicos ligados a la industria pesada, a través de grandes empresas públicas (o grupos nacionales) en los sectores siderúrgicos (acero) y los metalúrgicos (aluminio).

No obstante ello, la falta de articulación entre el subsistema científico, los distintos subsistemas tecnológicos y los incentivos que buscaban generar condiciones de protección a la industria naciente, se tradujo en la incapacidad de movilizar el conjunto de acciones hacia la reducción de la brecha tecnológica (Ferrer, 1974).

A finales de los años setenta, en el marco de un drástico proceso de apertura con apreciación cambiaria, se desmonta el conjunto de instituciones y agencias encargadas de implementar las promociones industriales. $\mathrm{Al}$ mismo tiempo, y de forma paradójica, se crea una amplia variedad de regímenes promocionales con foco regional, tales como Tierra del Fuego, Régimen Cuatro Provincias, y de Puertos Patagónicos (Azpiazu, 1987). En forma similar durante los ańos noventa, en el marco de la segunda experiencia general de apertura con apreciación cambiaria, se extiende la vigencia de los regímenes regionales y se implementan nuevos regímenes sectoriales como es el caso del de la industria automotriz ${ }^{4}$ (Filadoro, 2007; Cantarella et al., 2008; Lavarello \& Saller, 2007).

En forma adicional, una nueva etapa en la adición instrumentos se evidencia a partir del año 1996, con una nueva generación de políticas horizontales de apoyo a las PyMEs y las primeras acciones de apoyo a la generación de

\footnotetext{
Al mismo tiempo que propició una desgravación arancelaria para las importaciones de vehículos y sus componentes destinados a la producción local, a partir de 1994 se estableció un régimen que habilitaba a las terminales nacionales por un lado, establecer un esquema de intercambio administrado con Brasil, y a incorporar automotores fabricados hasta el 40-42\% del valor de los vehículos con autopiezas importadas, fijando un cronograma de reducción gradual que se aceleraría hacia el final del periodo.
} 
capacidades tecnológicas partiendo del reconocimiento de la existencia de asimetrías tecnológicas. ${ }^{5}$

En resumen, Argentina comienza para el ańo de 1976 un largo proceso de cambio de visión de política industrial que va desde un enfoque basado en la industrialización por sustitución de importaciones a un enfoque que combina la liberalización con la implementación de regímenes regionales y sectoriales, y hacia el final se les suman instrumentos basados en las políticas horizontales. $\mathrm{Al}$ igual que Brasil, y a diferencia de la experiencia asiática, este pasaje no se dio por reemplazo de instrumentos, sino por adición de capas geológicas, reforzando la gran debilidad en la implementación (Baruj et al., 2006; Lavarello \& Sarabia, 2015).

\section{EL (BREVE E INCOMPLETO) REGRESO DE LA POLÍTICA INDUSTRIAL A ARGENTINA A INICIOS DE LOS 2000: ASPECTOS CUANTITATIVOS}

Tras el colapso de la segunda experiencia de apertura con apreciación cambiaria, Argentina experimentó entre el año 2003 y 2013 una recuperación en la participación de la industria manufacturera en el valor agregado de la economía después de cinco años de caída sistemática. De esta manera comenzó a revertirse un ciclo de 26 años de desindustralización aumentando la participación de la industria manufacturera en el producto interno bruto (PIB) a precios corrientes de $18.5 \%$ promedio en el periodo $1995-2000$ a $21.7 \%$ promedio entre 2003 y 2012 (estimado con base en datos de la UNCTAD).

Estas evoluciones muestran que la industria manufacturera ha logrado resistir en términos agregados al proceso de desindustrialización que predominó en la región. Cabe preguntarse cuáles fueron las acciones de política económica que están detrás de este proceso de reindustrialización.

La primera evidencia del retorno de la política industrial es el aumento experimentado en los recursos fiscales y financieros orientados a la política industrial. A lo largo del periodo se evidencia un aumento sistemático del monto de los recursos fiscales y financieros asignados a la industria a partir de instrumentos o programas del sector público, acompañado por la ampliación de las áreas de intervención con el surgimiento de instrumentos más selectivos.

5

En el año de 1996, se crea la Agencia Nacional de Promoción Científica y Tecnológica bajo la órbita de la SECYT, autoridad de aplicación de la Ley 23.877 de promoción a las actividades científicas y tecnológicas.

http://unctadstat.unctad.org/EN/ 
El total de recursos fiscales y del sistema financiero asignados a la industria directa o indirectamente aumentaron del $0.9 \%$ del PIB en promedio para el subperiodo 2004-2006 al 1.4\% del PIB promedio en el periodo 2010-2013 (Lavarello \& Sarabia, 2015).

A fin de analizar los cambios en la configuración de las políticas siguiendo la grilla conceptual desarrollada en la sección 2, en el cuadro 1 se sistematizan estimaciones de la evolución de los recursos fiscales y financieros dedicados a la industria manufacturera entre el periodo 2004-2006 y 2010-2013 elaboradas en trabajos previos (Lavarello \& Sarabia, 2015).

Con la intensión de cuantificar los recursos, se hizo foco tanto en los gastos tributarios originados en regímenes de promoción económica regionales como en las asignaciones específicas de programas del Ministerio de Industria, el Ministerio de Ciencia,Tecnología e Innovación Productiva, el Ministerio de Planifcación y el Ministerio de Salud (véase Apéndice estadístico).

Un primer aspecto a reasaltar es que los incentivos fiscales horizontales prevalecieron como principal mecanismo de apoyo a la industria en un monto aproximado al $0.6 \%$ del PIB. Es de destacar que pese a su persistencia como mecanismo de subsidio al sector, los incentivos fiscales horizontales como las desgravaciones de exportaciones implementadas durante los años noventa disminuyen como contrapartida del mayor peso de los regímenes promocionales denotando la persistencia de las capas geológicas de diseños de política de los años setenta y ochenta.

Pese a la persistencia de las acciones dominadas por las capas geológicas, se produjeron algunos cambios en términos de política industrial y tecnológica. Este es el caso del incremento de los recursos en las políticas de apoyo a las oportunidades de la infraestructura de сут y a las capacidades tecnológicas de las firmas implementados desde la Secretaría de Ciencia y Tecnología (a partir del año 2008 rejerarquizada como Ministerio). Dicha intervención en la primera etapa de los años 2000 obedeció a un esquema de Estado facilitador que promoviera a partir de instrumentos horizontales la generación de capacidades en las empresas o el surgimiento de empresas tecnología intensivas. En particular, se destacan las políticas de apoyo a las capacidades tecnológicas en las firmas desde la Agencia Nacional de сут que pasan de $2.4 \%$ al $3.5 \%$ del total de recursos. Proceso que se ve acompañado por importantes aprendizajes institucionales que permiten avanzar de instrumentos horizontales a políticas selectivas por sector y tecnología.

A partir del ańo 2010, la política de apoyo selectivo a firmas muestra el segundo gran cambio del periodo con el subsidio con aportes del Tesoro a empresas estatales que buscan impulsar proyectos estructurantes desde un in- 
cipiente enfoque orientado por misión (mission oriented). En el marco de estos proyectos se financió la generación de capacidades de un conjunto de empresas públicas y proveedores asociados a los sectores aeroespacial, nuclear y de defensa, aumentando los recursos de $0.01 \%$ al $0.14 \%$ del piB total (y de $0.2 \mathrm{al}$ $1 \%$ del PIB manufacturero), lo que representa el $9.8 \%$ del total de los recursos orientados a la industria.

Finalmente, un tercer aspecto a destacar es el impulso que asume a partir del ańo 2010 la política de financiamiento a partir de la reforma de la Carta Orgánica del Banco Central de la República Argentina (BCRA). Pese a que los nuevos instrumentos se orientaron a financiar nuevas inversiones en plantas industriales o a fortalecer las capacidades productivas en las PyMEs, los mismos no tuvieron como prioridad la generación de capacidades tecnológicas, sino que tuvo un claro objetivo anti-cíclico en un contexto de desaceleración macroeconómica. Por ello, estos instrumentos fueron mutando el objeto de crédito desde el financiamiento a la formación de capital al financiamiento del capital de trabajo desvinculado de toda estrategia de industrialización o reducción de brecha tecnológica.

\section{HACIA UNA PERIODIZACIÓN DE LA POLÍTICA INDUSTRIAL DE LOS AÑOS 2000}

De la revisión de los esfuerzos fiscales y financieros de apoyo a la industria surge como interrogante si dicho mayor esfuerzo estuvo asociado a la multiplicación de visiones y problemas a los que se fue dando respuesta en forma parcial o emergió, ya sea en forma explícita o implícita, una coordinación de acciones que superen la visión de la política industrial como la respuesta reactiva a fallas de mercado.

\subsection{El subperiodo 2004-2006: los derechos de exportación como instrumento de política industrial}

Paradójicamente, el principal instrumento que operó sobre los incentivos desafiando las ventajas comparativas no fue estrictamente un instrumento de política industrial, sino de política macroeconómica. En el periodo se implementó un esquema de derechos de exportación diferenciados favoreciendo con derechos menores a la industria vis à vis a las actividades primarias $\mathrm{y}$ agroindustriales. 


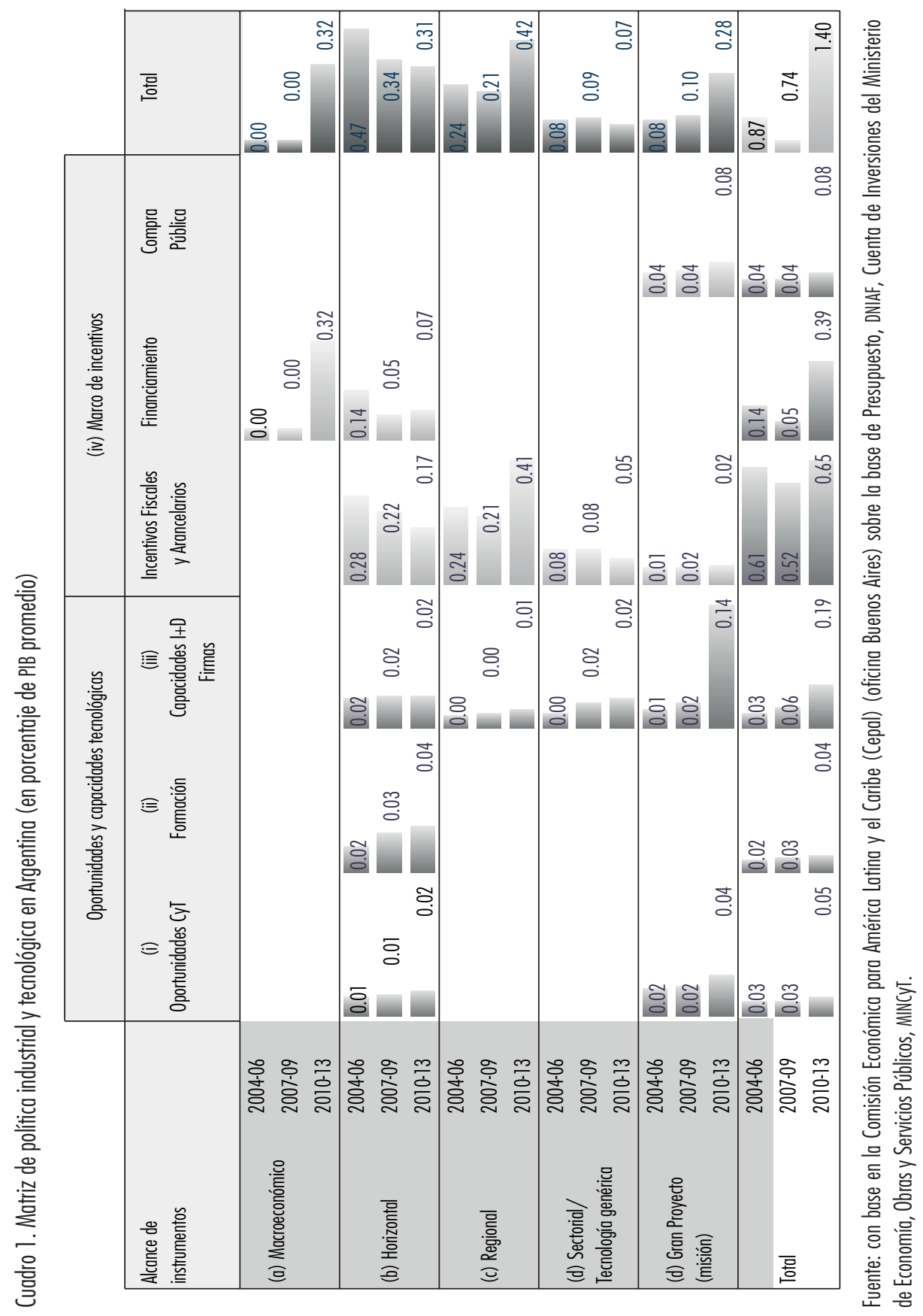


Este mecanismo fue el principal -si no el único- que buscó afectar los incentivos desafiando las ventajas comparativas vía señales de precios. De allí que el mismo debe ser considerado parte central de la experiencia de política industrial de los tempranos ańos 2000. Dicho mecanismo, junto al impulso sobre la demanda efectiva a partir de una serie de medidas reparadoras de la situación socio-laboral, contribuyeron a aumentar el peso del valor agregado de las actividades manufactureras de origen industrial.

Para ilustrar la incidencia de este tipo de instrumento, se puede estimar la alícuota promedio implícita en los datos de recaudación por derechos de exportación y el valor de las exportaciones para distintos periodos. Como se evidencia en forma estilizada en la gráfica 1, las alícuotas incorporadas a los derechos de exportación de la industria manufacturera de origen industrial (MOI) resultaron, de manera significativa, inferiores a las alícuotas respectivas de los sectores con los que el país tuvo ventajas comparativas naturales, como fueron los sectores primarios y más aún las manufacturas de origen agropecuario (MOA).

Gráfica 1. Derechos de exportación: alícuotas implíitas por rubro económico

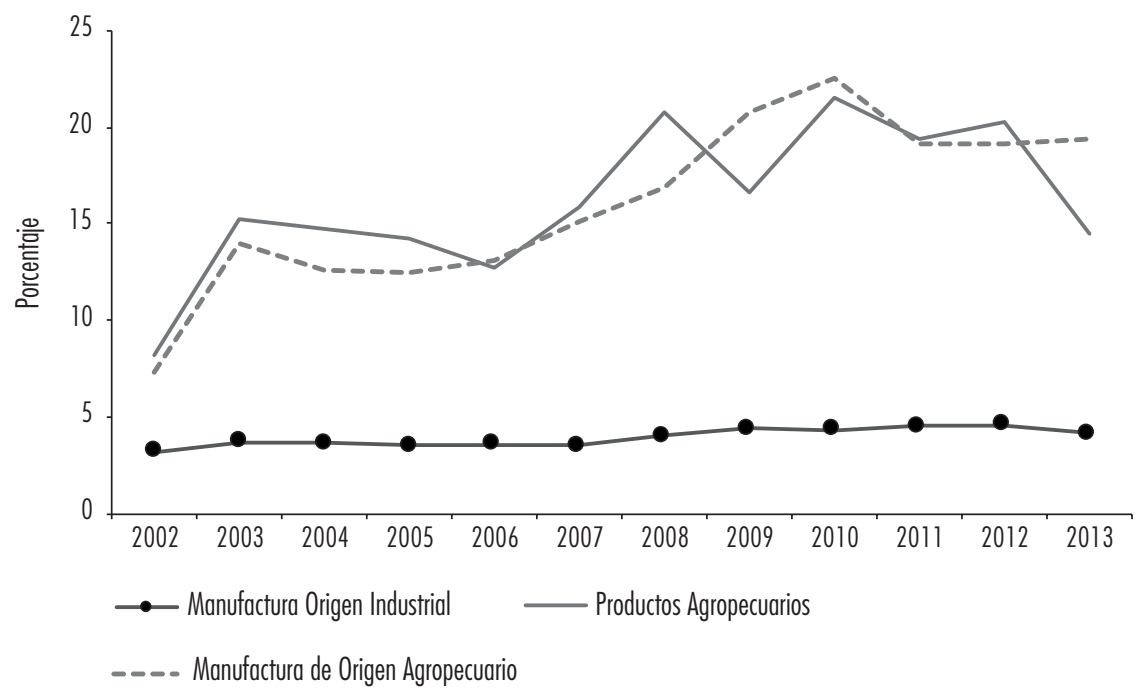

Fuente: Lavarello y Mancini (2017) con base en la Comisión Económica para América Latina y el Caribe (Cepal) (oficina Buenos Aires) sobre la base de presupuesto, DNIAF, Cuenta de Inversiones del Ministerio de Economía, Obras y Servicios Públicos, MINCyT. 
Más allá del tipo de cambio competitivo con derechos de exportación diferenciales, las desgravaciones y los modos impositivos de diferir los pagos asociados a los regímenes de promoción regional vigentes desde la década de los ochenta continuaron siendo los principales instrumentos en términos de recursos fiscales involucrados. Este tipo de mecanismo, cuyo ejemplo más emblemático es el régimen de Tierra del Fuego, enfrentó desde su creación a inicios de los años ochenta, importantes problemas de diseño e implementación, asociados al bajo grado de monitoreo asociado a su diseño institucional (Filadoro, 2007).

Entre las capas geológicas de instrumentos de políticas económicas, además de los regímenes regionales, sobresalió el régimen automotriz; implementado durante los años noventa tuvo un rol crucial en la adecuación de la operatoria de las filiales de las empresas multinacionales a los cambios impulsados desde sus casas matrices. A partir de la segunda mitad de los años noventa se buscó reformular el régimen para incluir a los autopartistas, pero dicha inclusión se tradujo en una protección arancelaria negativa y el establecimiento de requisitos de integración nacional aún menores que los que tenían en la práctica las filiales. Desde inicio de los años 2000 se implementaron un conjunto de incentivos fiscales buscando paliar sin éxito los efectos negativos del mencionado régimen sobre sector autopartista (Lavarello \& Saravia, 2015).

Sin embargo, los nuevos incentivos fiscales no logran reparar la estructura de un sector en el que la configuración del sector autopartista a nivel regional limita la producción en el país a ciertos componentes periféricos en los que existen proveedores altamente competentes (válvulas, correas, etcétera) y componentes "sistémicos" de alto costo de transporte (guardabarros, asientos, etcétera). Salvo excepciones puntuales como las transmisiones y los ensambles de motores con algún grado de integración local en marcas particulares, los conjuntos y subconjuntos más complejos se importan de Brasil.

Durante este periodo también surgen nuevos instrumentos de promoción sectorial que se suman al régimen automotriz. En particular, se creó un régimen de promoción a la producción de bienes de capital, informática, telecomunicaciones y maquinaria agrícola. Este mecanismo tuvo escasa efectividad en la medida que la obtención del subsidio no fue acompañado de requisitos de desempeño y mecanismos de monitoreo lo que limitó las posibilidades de sustitución de importaciones en componentes intensivos y en tecnología (Lavarello \& Goldstein, 2011; Peirano, 2013).

Luego, en el marco de la inercia de los regímenes regionales y sectoriales previos paradojalmente fue la política macroeconómica la principal herramienta de apoyo a la industria. Instrumento que fue compensado por el 
impulso a la demanda efectiva asociado a un conjunto de medidas reparadoras de la situación social que dejó la experiencia de apertura de los años noventa.

\subsection{El subperiodo 2007-2009: reforzamiento de las capacidades tecnológicas}

En este subperiodo maduran importantes aprendizajes institucionales a partir de políticas horizontales de fomento a las capacidades tecnológicas y de apoyo a las PyMEs implementados en la década previa. En el año 2008 posibilita el diseño de una nueva gama de instrumentos selectivos de apoyo a las capacidades tecnológicas en nuevos paradigmas tecnológicos (software, nanotecnología y biotecnología), al mismo tiempo que se inicia el programa de fortalecimiento de la educación técnica y de la enseñanza de ingeniería con una clara orientación hacia el sector manufacturero.

Si bien durante el periodo Argentina continua presentando esfuerzos tecnológicos menores que los países desarrollados, es a partir de mediados de los ańos 2000 que el gasto en I+D y la cantidad de ingenieros aumenta sensiblemente. De acuerdo a estadísticas compiladas por la RICyт, ${ }^{7}$ durante los años noventa el gasto en I+D representó el $0.38 \%$ del pIB promedio durante los años noventa, y alcanzó el $0.47 \%$ promedio entre el año 2003 y 2010. De la misma forma el número de ingenieros se incrementó de 1.5 en 1990 a 2.6 de cada 10 mil habitantes en el año 2013.

Estas acciones obedecen a políticas sobre las oportunidades y capacidades tecnológicas implementadas en el periodo. En materia de apoyo a las capacidades tecnológicas de las firmas se evidencia un pasaje desde instrumentos horizontales hacia instrumentos por tecnología transversal y por aglomerados productivos.

Entre los proyectos con mayor grado de avance e impacto en la generación de un entramado productivo, sobresale el desarrollo del clúster de maquinaria agrícola de la región centro de Santa Fe y Córdoba (Lavarello \& Goldstein, 2011). En línea con el aprendizaje institucional, dicho clúster contaba con una trayectoria de asistencia técnica regional al sector en cuestión (superior a los 10 años) (Moori Koenig, 2010).

Red de Indicadores de Ciencia y Tecnología -Iberoamericana e Interamericana- (RICyT). S www.ricyt.org 
El mismo fue resultado del aprendizaje institucional previo de la Agencia Nacional de Política Científica y Tecnológica, y permitió una mayor articulación entre oferta y demanda de conocimiento, integrando distintas líneas de apoyo de generación de capacidades tecnológicas de y aquellas de financiamiento a la ciencia y del financiamiento a la tecnología.

\subsection{El subperiodo 2009-2015: el Estado como productor y usuario en un contexto macroeconómico adverso}

Frente a la erosión del tipo de cambio como mecanismo de incentivo a la industria en el marco de una creciente incertidumbre asociada a la crisis internacional, surgen nuevos mecanismos de protección basados en la administración del comercio y la reaparición del Estado como productor y usuario bajo una nueva modalidad de intervención orientada por misiones.

En materia de generación de oportunidades tecnológicas se inició, por un lado, un conjunto de proyectos tecnológicos en áreas de $\mathrm{I}+\mathrm{D}$ en tecnologías satelitales, energéticas y de defensa bajo un esquema "desde arriba" y, por el otro, se avanzó en una reorientación (parcial) de los fondos de apoyo a la сут orientados al fortalecimiento de capacidades tecnológicas con un fuerte peso de las actividades biotecnológicas. Estos apoyos pasaron de 3\% del total de recursos en el periodo 2004-2006 en financiamiento de la I+D aplicada a cerca de 5\% en el periodo 2010-2013.

Es de destacar el mayor peso de acciones estructuradas según misiones en grandes proyectos estatales con potencial de traccionar al sector privado, ya sea a partir de la compra estatal o el financiamiento directo a empresas estatales. Los recursos de estos programas pasan de 0.3 al $1.7 \%$ del valor agregado industrial y explican un aumento de la participación de 7.7 a más de 13\% del total de apoyos. La recuperación de YPF, los grandes proyectos satelitales, nucleares, de defensa y compras gubernamentales en el sector salud marcando una nueva etapa de política industrial que se acelera a partir del año 2010.

Existe un conjunto de instrumentos cuya importancia no se puede captar a partir de los recursos fiscales. Este es el caso ya mencionado de los esquemas de tipo de cambio diferenciales y, más tarde, la recuperación de las políticas de comercio administrado a través de distintos mecanismos no tarifarios -primero las licencias no automáticas (LNA) de importaciones y luego las Declaraciones Juradas Anticipadas de Importación (DJAI) - permitieron recuperar las capacidades del Estado para llevar adelante mecanismos de protección selectivos. Desde el año de 2012, las DJAI reemplazarían de manera paulatina a 
las LNA. Pese a las dificultades iníciales asociadas a la ausencia de capacidades institucionales, el instrumento se fue adaptando y posibilitó mecanismos de interacción con el sector privado acordando metas de producción, empleo e inversión con el posterior monitoreo por parte de la autoridad de aplicación. ${ }^{8}$ Luego, aún si la implementación de los mecanismos no tarifarios implicaron adaptaciones sucesivas en respuesta a los condicionamientos de la Organización Mundial de Comercio (OMC), la política económica recuperó durante el periodo la capacidad de intervenir en forma deliberada en las condiciones de selección de mercado.

\subsection{La trayectoria de política industrial a lo largo de los subperiodos}

El análisis de los distintos subperiodos permite inferir una trayectoria de intervención que partiendo de una modalidad de Estado regulador avanzó hacia un rol más activo en el que la política industrial reaparece bajo una modalidad de Estado facilitador. El regreso de la política industrial se expresa bajo esta nueva modalidad en el caso del plan estratégico industrial PEI 2020 en el año de 2011.

No obstante ello, es posible apreciar a partir del año de 2011 el pasaje de un Estado facilitador a uno en el que se comienzan a promover capacidades tecnológicas infantiles de forma más selectiva, según tecnología o sector, que se refuerza con la creación, renacionalización o fortalecimiento de empresas en el área satelital, energía y nuclear. La aparición de un abordaje de grandes proyectos estructurados por misión sería la última modalidad de intervención.

Este pasaje del Estado facilitador a una modalidad de intervención que promueve las capacidades infantiles, no llega a estructurarse en un plan con jerarquía institucional que integre distintos instrumentos y acciones. Límite que se tradujo en una aún débil articulación entre los instrumentos de apoyo a las capacidades tecnológicas, los diversos mecanismos de incentivos preexistentes y la promisoria recuperación de las políticas de financiamiento (véase figura 2).

En el año de 2014 se realizaron 633 acuerdos con grandes empresas, que representaban USD 52.491 millones de importación en ese año, y 3.086 con PyMEs por USD 4.677 millones (en 2015 se sumaron otras 1500 ). En total, excluidas las compras al exterior de combustibles, aviones y trenes, el nuevo régimen de administración del comercio abarcó el $87 \%$ de las importaciones de 2014 (se estima que habría alcanzado el $90 \%$ en 2015) con un impacto sobre el ahorro de divisas de USD 1.230 millones, excluyendo al sector automotor y de electrónica. 
Figura 2. Política industrial en Argentina: distintas trayectorias institucionales

\begin{tabular}{|c|c|c|c|c|c|c|}
\hline \multirow[b]{2}{*}{$\begin{array}{l}\text { Alcance de } \\
\text { instrumentos }\end{array}$} & \multicolumn{3}{|c|}{ Oportunidades y capacidades tecnológicas } & \multicolumn{3}{|c|}{ (iv) Marco de incentivos y contexto de selección } \\
\hline & $\begin{array}{l}\text { (i) } \\
\text { Oportunidades } \\
\text { Cy厂 }\end{array}$ & $\begin{array}{l}\text { (ii) } \\
\text { Aprendizajes } \\
\text { distribuidos }\end{array}$ & $\begin{array}{c}\text { (iii) } \\
\text { Capacidades } \\
\text { I + D Firmas }\end{array}$ & $\begin{array}{l}\text { Incentivos } \\
\text { fiscales y } \\
\text { arancelarios }\end{array}$ & $\begin{array}{l}\text { Administración } \\
\text { de comercio }\end{array}$ & $\begin{array}{l}\text { Compra } \\
\text { pública }\end{array}$ \\
\hline $\begin{array}{l}\text { (a) Macro- } \\
\text { económico } \\
\text { (b) Horizontal }\end{array}$ & \multicolumn{3}{|c|}{$\begin{array}{c}\text { Estado facilitador } \\
\text { 97-06: Políticas Cientificas y Tecnológicas } \\
\text { Horizontales (foncy, Fontar) }\end{array}$} & \multirow{2}{*}{\multicolumn{3}{|c|}{$\begin{array}{l}\text { '90: Devaluaciones fiscales, reintegro a las } \\
\text { exportaciones, Defensa competencia, Privatización } \\
\text { Regímenes Tierra del Fuego Automotriz, etcétera }\end{array}$}} \\
\hline \multirow{2}{*}{ (c) Regional } & & $\downarrow$ & & & & \\
\hline & & $\begin{array}{l}\text { 2008: Fonarsec Bio, } \\
\text { 2014: Fondos Se }\end{array}$ & $\begin{array}{l}\text { ss, Nano } \\
\text { oriales }\end{array}$ & $\begin{array}{l}\text { 2: Tipo de cambio } \\
\text { diferenciales }\end{array}$ & $\begin{array}{c}2009-13 \text { Control } \\
\text { Importaciones }\end{array}$ & \\
\hline (d) Sectorial/ & & & OMOCIÓN CAPAC & DADES INFANTILE & & \\
\hline $\begin{array}{l}\text { (d) Orientado } \\
\text { por misión }\end{array}$ & & & $\begin{array}{r}\text { MIS } \\
\text { Campeon } \\
\text { (YPF, NUCLEAR, }\end{array}$ & $\begin{array}{l}\text { NES } \\
\text { estatales } \\
\text { SAT, Defensa) }\end{array}$ & & \\
\hline
\end{tabular}

Fuente: elaboración propia.

\subsection{Las primeras iniciativas de articulación de instrumentos: los planes industriales y de innovación en Argentina}

Entre los años 2010 y 2015 surgieron dos iniciativas de planificación de política industrial y tecnológica desde distintas áreas del Estado (véase figura 3). La primera fue el Plan Estratégico Industrial 2020, cuya génesis obedeció a los límites que fue enfrentando el proceso de crecimiento asociado a la restricción externa.

La definición de los lineamientos del PEI, otorgados en un proceso participativo, partió de la necesidad de identificar "cuellos de botella" en cada cadena (Stumpo \& Rivas, 2013). Para tal fin, se convocaron a 11 foros con representantes del sector productivo, los ministerios nacionales y/o provinciales, y los ámbitos académicos y de formación vinculados a la investigación aplicada. Dichos foros dieron lugar a la conformación de 11 mesas de implementación que participarían en el seguimiento y en la identificación de nuevos "cuellos de botella” en su desarrollo. 
Figura 3. Iniciativas de planificación de la políitica industrial y tecnológica
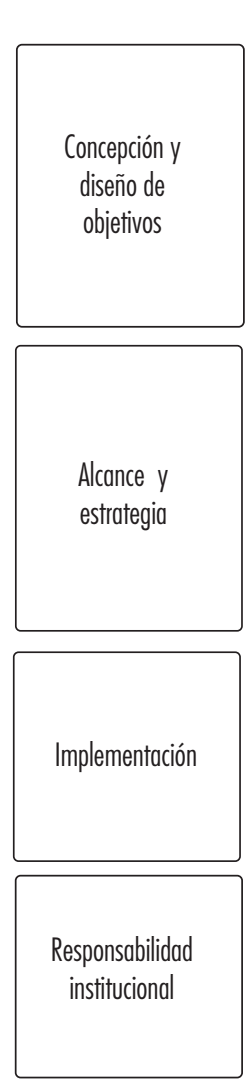

PLAN ESTRATÉGICO INDUSTRIAL 2020

Objetivo general: dar sustentabilidad al proceso de crecimiento.

Pre-selección de 11 cadenas: perspectiva horizontal de política (no hay cadenas prioritarias) $\rightarrow 11$ foros (1 por cadena).

- General sustitución de importaciones a partir de las 11 cadenas, viraje hacia desarrollo e integración local.

- Estado "facilitador": problemas de coordinación por asimetrías de información entre la oferta de instrumentos y el sector privado.

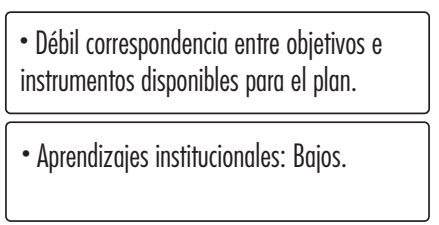

- La subsecretaría encargada de la
implementación acompaña el proceso (sin
recursos propios).
- Mayor integración inter-ministerial
(formal).

PLAN ARGENTINA INNOVADORA 2020

Objetivo general: impulsar la innovación productiva sobre la base de las oportunidades CyT existentes.

35 "Núcleos Socio-Productivos": articulan tecnologías, sectores y regiones. Mesas de trabajo (sólo cámaras y academia).

- Focalización capacidades tecológicas sectoriales (SALUD, AGROINDUSTRIA, ENERGÍA, AMBIENTE , en menor medida en Metealmecánica y Electrónica)

- Débil apoyo a sector bienes de capital y grandes proyectos (nuclear, aeroespacial).

- Mayor correspondencia entre objetivos e instrumentos (FS).

- Aprendizajes institucionales: creciente selectividad ex ante.

- La subsecretaría encargada de la implementación acompaña el proceso (con acceso gradual a recursos).

- Débil integración inter-ministerial (exc. Secretaría de Agricultura).

Fuente: elaboración propia.

Este plan se caracterizó por su alta generalidad en la selección sectorial abarcando a la totalidad de cadenas manufactureras. Pese al carácter participativo del mismo, la baja correspondencia entre la ambición del plan y los recursos afectados limitó su alcance efectivo. El éxito de una u otra mesa de implementación dependió no únicamente de la priorización ex ante de los sectores desde una perspectiva estratégica, sino de la composición y fortaleza de la institucionalidad del sector privado. 
En forma simultánea, los aprendizajes institucionales del міNсут dieron lugar al plan Argentina Innovadora 2020. El plan presentó una mayor correspondencia entre objetivos, instrumentos y la capacidad de coordinación e implementación que el Plan Estratégico Industrial (PEI). En este caso se avanzó hacia una mayor selectividad de las acciones e instrumentos, definiendo 35 "núcleos socio-productivos estratégicos" que combinaban la selección de un sector, una región y una base tecnológica.

Uno de los principales límites que enfrentó este plan es la baja articulación con otras áreas de política industrial y tecnológica que se manifestó particularmente con el PEI 2020 y con los desarrollos estatales de satélites y aplicaciones de defensa, en los que el Estado asumió un rol empresario en el periodo.Esta modalidad de intervención no permitió generar ciertos polos movilizadores de distintas acciones de política industrial.

En ambos casos, la falta de una instancia coordinadora que cuente con la jerarquía necesaria y las capacidades institucionales como para alinear los distintos instrumentos en función de metas sectoriales o misiones implicó que, a excepción de ciertos sectores en los que se tiene una institucionalidad público-privada desarrollada, las metas de estos planes no se tradujeran en acciones y resultados.

\section{REFLEXIONES FINALES Y TRAYECTORIAS POSIBLES DE POLÍTICA INDUSTRIAL}

El trabajo muestra que, a pesar de la inercia de las "capas geológicas" de los regímenes de promoción regional precedentes, y sin llegar a conformar una política industrial integral, en el periodo 2003-2015 se implementó un conjunto de instrumentos e instituciones orientados a generar oportunidades y capacidades tecnológicas, financiamiento y mecanismos de selección, que dieron lugar a un regreso incipiente aunque incompleto de la política industrial.

- En materia de generación de oportunidades y la promoción de capacidades tecnológicas se inició, por un lado, un conjunto de proyectos en áreas de $\mathrm{I}+\mathrm{D}$ en tecnologías satelitales, energética y de defensa bajo un esquema "desde arriba" en el que los ministerios de Planificación y de Defensa jugaron un rol central impulsando distintos organismos públicos. Por otro lado, se avanzó en una mayor selectividad sectorial de los fondos de apoyo a la сут del мincyт. 
- Por su parte, se llevaron adelante un conjunto de instrumentos cuya importancia no se puede captar a partir de los recursos fiscales destinados al sector. Este es el caso ya mencionado de los esquemas de tipo de cambio diferenciales y más tarde la recuperación de las políticas de comercio administrado a través de distintos mecanismos no tarifarios (LNA, DJAI), que pudieron permitir al Estado recuperar las capacidades para llevar adelante medidas de protección selectiva.

- Estos avances no fueron acompañados por la una instancia superior de implementación de la política en la que los planes se correspondan con los recursos y la Agencia (o Secretaría) cuente con la jerarquía institucional necesaria. Ausencia que se traduce en una débil coordinación entre las distintas áreas de intervención e instrumentos, limitando las posibilidades de éxito de toda experiencia de aprendizaje tecnológico en el marco de una estrategia de industrialización.

Esta falencia en materia de implementación se ve reforzada por la dificultad de establecer precios relativos que favorezcan a los sectores dinámicos en un país en los que los sectores tradicionales vinculados a la producción de materias primas básicas cuentan con poder de veto sobre la dirección de la política económica. La política industrial en países en desarrollo requiere tener en cuenta la necesidad de acciones deliberadas desde el Estado, para lo cual se requiere una unidad de comando del proceso para no terminar reproduciendo la estructura productiva preexistente.

Luego es posible sostener que si bien Argentina asiste de manera parcial a la aparición (o revalorización) de un conjunto de acciones que promovieron la industrialización a partir de acciones e instrumentos gradual, pero crecientemente más selectivos. No obstante ello, este conjunto de acciones reflejaron bajos grados de coordinación. En este sentido si bien el regreso de la política industrial implicó importantes aprendizajes institucionales, también fue incompleto y transitorio.

Con el cambio de gobierno hacia fines del año de 2015, se inició un proceso de revisión de las acciones en términos de incentivos y protección selectiva que favorecían a la industria manufacturera. Se suprimió el esquema de derechos de exportación diferenciales con el consecuente restablecimiento de las "señales correctas" de mercado. En el marco del ajuste presupuestario, los grandes proyectos estructurantes comenzaron a ser rediscutidos buscando una mayor participación de capital extranjero. Los equipos técnicos de las agencias u organismos de implementación que más aprendizaje habían 
logrado fueron suprimidos o reasignados a otras áreas. Al mismo tiempo los viejos esquemas promoción regional se combinaron con una gradual apertura de importaciones.

\section{APÉNDICE ESTADÍSTICO}

A fin de estimar los recursos públicos asignados en forma directa o indirecta, para promover y/o sostener actividades vinculadas a la producción manufacturera local, se sistematizó la información con base en instrumentos con variables clave, tales como: área de intervención, tipo de política, tipo de beneficio y alcance-horizontal, sectorial o regional-.

Los gastos tributarios originados en regímenes de promoción económica y las asignaciones específicas de programas del Ministerio de Industria y del Ministerio de Ciencia, Tecnología e Innovación Productiva (мІмсут) constituyeron el eje; se priorizaron asignaciones según:

1. Información específica sobre el monto ejecutado y/o desembolsado de cada programa, siempre que fuera pública y estuviera sistematizada (Fondo $\mathrm{Na}$ cional para el Desarrollo de la Pequeña y Mediana Empresa -Fonapyme-, Fondo Tecnológico Argentino-FONTAR-, etcétera).

2. La cuenta de inversión, cuando contemplara el gasto ejecutado por programa. La serie del régimen de promoción de software, por ejemplo, corresponde a los gastos tributarios, a excepción de 2011-2013 que se basa en la cuenta de inversión por grandes discrepancias estadísticas.

3. Las partidas asignadas por la ley anual de presupuesto, ajustadas por el porcentaje de ejecución de cada programa, conforme a la cuenta de inversión.

\section{BIBLIOGRAFÍA}

Abeles, M., Lavarello, P. \& Montagu, H. (2013),"Heterogeneidad estructural y restricción externa en la economía argentina”, en R. Infante, Hacia un desarrollo inclusivo. El caso de la Argentina, Cepal, 23-95.

Amsden, A. H. (1992), Asia's Next Giant: South Korea and Late Industrialization, Oxford, University Press.

Azpiazu, D. (1987), "Los resultados de la política de promoción industrial al cabo de un decenio (1974-1983)”, Desarrollo Económico, 631-651. 
Azpiazu, D. y Nochteff, H. (1995), El desarrollo ausente: restricciones al desarrollo, neconservadorismo y elite económica en la Argentina. Ensayos de economía politica, Tesis grupo editorial Norma.

Baruj, G. y Porta, F. (2006), "Políticas de competitividad en la Argentina y su impacto sobre la profundización del Mercosur", Documento de proyecto núm. 93, Cepal, Naciones Unidas, Santiago de Chile.

Bell, M. \& Pavitt, K. (1995), The Development of Technological Capabilities. Trade, Technology and International Competitiveness, 22 (4831), 69-101.

Bisang, R., Bonvecchi, C., Kosacoff, B. \& Ramos, A. (1996), "La transformación industrial en los noventa. Un proceso con final abierto", Desarrollo Económico, 187-216.

Braude, H., Dragún, P., García, M. Ginsberg, M. \& Tavosnanska, A. (2009), “La política industrial argentina 2003-2008. Transitando por los suburbios de la micro", Congreso Anual de Aeda, Buenos Aires.

Cantarella, J., Katz, L. \& de Guzmán, G. (2008), "La Industria Automotriz Argentina: limitantes a la integración local de autocomponentes", DT 01/2008, Laboratorio de Investigación sobre Tecnología, Trabajo, Empresa y Competitividad (LITTEC), Instituto de Industria, Universidad Nacional de General Sarmiento.

Cepal (2011), "Lineamientos estratégicos para el desarrollo industrial en Argentina: documento de base", Cepal, Oficina de Buenos Aires.

Ciecti (2014), "Asociatividad para la innovación con alto impacto sectorial. Congruencia de objetivos entre las áreas programática y operativa de los Fondos Sectoriales", Documento de trabajo, мілсут, Ciudad Autónoma de Buenos Aires.

Cimoli, M., Dosi,G., Nelson, R.\& Stiglitz, J. (2006), "Institutions and Policies shaping Industrial Development: An Introductory Note", en Cimoli, M., Dosi, G. \& Stiglitz, J. (coord.), Industrial Policy and Development: The Political Economy of Capabilities Accumulation, Oxford, University Press.

Cimoli, M. \& Rovira, S. (2008), "Elites and Structural Inertia in Latin America: An Introductory Note on The Political Economy of Development", Journal of Economic Issues, 327-347.

Cimoli, M., Ferraz, J. \& Primi, A. (2009), "Science, Technology and Innovation Policies in Open Global Economies: Reflections from LAC", GCG Geortown University-Universia, vol. 3, núm. 1.

Cohen, E. (2007), "Industrial Policies in France: The Old and The New", Journal of Industry, Competition and Trade, 7 (3-4), 213-227. 
Crespi, G., Fernández-Arias, E., \& Stein, E. (2014). Rethinking productive development. In Rethinking Productive Development (3-31), Palgrave Macmillan US.

Evans, P. (1995), Embedded Autonomy: States and Industrial Transformation, Princeton, Princeton University Press.

Ferrer, A. (2014), Tecnología y politica en América Latina, Bernal, Editorial de la Universidad Nacional de Quilmes y AEDA.

Filadoro, A. (2007), "Impacto de la promoción en la estructura de la industria de Tierra del Fuego", en Lavarello P. \& Saller G. (coord.), Promoción industrial y regional: efectos fiscales y casos paradigmáticos, IEFE.

Gutman, G. \& Lavarello, P. (2016), "Política industrial en el sector biofarmacéutico en la Argentina durante los años 2000", Cepal, Mimeo.

Hamilton, A. (1791) [2007]. Report on the Subject of Manufactures, Cosimo, Inc.

Hausmann, R. \& Rodrik, D. (2002), "Economic Development as Self-discovery", Working Paper num. 8952, National Bureau of Economic Research.

Heyn, I. \& Moldován, P. (2010), "La política comercial en las Estructuras Productivas Desequilibradas: el caso de las licencias no automáticas de importación”, en Chena, P. I, N. E. Crovetto \& D. Panigo, Ensayos en honor a Marcelo Diamand, Miño y Ávila editores, Buenos Aires.

Hirschman, A. (1958), "Enfoque generalizado del desarrollo por medio de eslabonamientos, con especial referencia a los productos básicos", Trimestre Económico, 1, 177-99.

Johnson, C. (1982), "MiтI and the Japanese Miracle", Stanford, Stanford University Press.

Katz, J. \& Kosacoff, B. (1998), “Aprendizaje tecnológico, desarrollo institucional y la microeconomía de la sustitución de importaciones", Desarrollo Económico, 483-502.

Lall, Sanjaya (1992), “Technological Capabilities and Industrialization”, World Development, 20(2), 165-186.

Lavarello P. Y Mancini M. (2017) "Politica Industrial y recuperación manufacturera en Argentina” en Cimoli, M., Castillo, M., Porcile, G., \& G. Stumpo (editores), Politicas Industriales y tecnológicas en America Latina, Cepal (en Prensa).

Lavarello, P. \& Saller, G. (2007), Promoción industrial y regional: efectos fiscales y casos paradigmáticos, IEFE.

Lavarello, P. \& Goldstein, E. (2011), "Dinámicas heterogéneas en la industria de maquinaria agrícola argentina”, Problemas del Desarrollo, vol. 42, núm. 166, México, unam-IIEC, 85-109. 
\& Sarabia, M. (2015), "La política industrial en la Argentina durante la década de 2000", Serie Estudios y Perspectivas, núm. 45, Buenos Aires, Cepal.

Lin, J. Y. \& Monga, C. (2011), "Growth Identification and Facilitation: The Role of The State in The Dynamics of Structural Change", World Bank Policy Research Working Paper Series, vol. 5313.

Lin, J. Y. (2012), New Structural Economics: A Framework for rethinking Development, World Bank Res Obs (2011) 26 (2): 193-221.

List, F. (1841) [1856], National System of Political Economy, JB Lippincott \& Company.

Mazzucato, M. (2013), "The entrepreneurial state: Debunking the public vs. private myth in risk and innovation", Anthem, London.

Moori Koeing, V. (2010), "Políticas de articulación productiva en Argentina. Análisis de los programas basados en clústers y cadenas productivas", en C. Ferraro (ed.), Clusters y politicas de articulación productiva en América Latina, Cepal-Fundes.

MTEyss (2010), “Trabajo y empleo en el Bicentenario: cambio en la dinámica del empleo y la protección social para la inclusión”, Proyecto PNUDAGR/04/034.

Pavitt, K. (1984), Sectoral Patterns of Technical Change: Towards a Taxonomy and a Theory, Research Policy, 13 (6), 343-373.

Peirano, F. (2013), "El complejo productivo de bienes de capital", en Stumpo, G. \& D. Rivas (coord.), La industria argentina frente a los nuevos desafios y oportunidades del siglo XXI, Santiago, Cepal.

Peres, W. \& Primi, A. (2009), "Theory and Practice of Industrial Policy. Evidence from the Latin American Experience”, ECLAC, Santiago, Chile, LC/L.3013-P.

Stumpo, G. \& Rivas, D. (2013), La industria argentina frente a los nuevos desafios y oportunidades del siglo XXI, Cepal.

Wade, R. (2014), "The Mystery of US Industrial Policy: The Developmental State in Disguise”, en J. M. Salazar Xirinachs \& R. Kozul Wright (eds.), Transforming Economies: Making Industrial Policies Work for Growth, Jobs and Development, 379-400, Geneva: ILO-UnCTAD. 
